\title{
The Association of Chronic Obstructive Pulmonary Disease and Osteoporotic Fracture in Older Patients
}

\author{
Qian Hong $\mathbf{W u}^{1}$ and Gwo Ping Jong ${ }^{2 *}$ \\ ${ }^{1}$ Department of Tuberculosis, Prevention and Treatment Hospital of Shaanxi province, China \\ ${ }^{2}$ Division of Internal Cardiology, Chung Shan Medical University Hospital and Chung Shan Medical University, Taiwan
}

Received: November 06, 2017; Published: November 14, 2017

*Corresponding author: Gwo-Ping Jong, Division of Internal Cardiology, Chung Shan Medical University Hospital and Chung Shan Medical University, and Basic Science, Central Taiwan University of Science and Technology, Taichung, Taiwan, Tel: 886-933450449; Fax: 886-424729993;

Email: cgp8009@yahoo.com.tw

\begin{abstract}
Recent epidemiological studies have demonstrated a close association between osteoporotic fractures and common chronic diseases such as chronic obstructive pulmonary disease (COPD), hypertension, chronic kidney disorาders, and diabetes. COPD is a chronic inflammatory airway disease, and it well known that osteoporotic fractures are common in patients with COPD. However, reports on the association between COPD and osteoporotic fractures have been inconsistent in the literature. The objective of this review is to discuss the existing literature on the potential association between COPD and osteoporotic fractures in older adults. A PubMed search was conducted to identify studies evaluating the potential association between COPD and osteoporotic fractures in older adults. Overall, the results of these studies showed a non beneficial effect. COPDs have been associated with an increased risk of osteoporotic fractures. Further research is needed on algorithms to identify COPD patients with a high risk of osteoporotic fractures and the beneficial effects of preventing osteoporotic fractures in older patients with COPD.
\end{abstract}

Keywords: Chronic obstructive pulmonary disease; Osteoporosis fracture; Mechanism

\section{Introduction}

Chronic obstructive pulmonary disease is the most common chronic respiratory disease in the world and results in increasing economic costs [1]. The prevalence of osteoporotic fractures, which is more common in older patients than in younger patients, has been estimated to be 9 million worldwide in 2000 [2]. By 2030, COPD has been projected to be the third leading cause of death caused by an increase in cigarette smoking $[3,4]$. Hence, concerns about the health care of COPD, particularly in terms of the related comorbidity with osteoporotic fractures, have gradually increased worldwide. Recently, several observational studies have opened a debate about whether COPD is associated with osteoporotic fractures in treated COPD patients [5-8]. A cross-sectional study conducted by Watanabe et al. reported that the prevalence of vertebral osteoporotic fractures is as high as 79.4\% in Japanese men with COPD [5]. Similarly, a nationwide population-based cohort study conducted by Lee et al. on 44,812 patients with a depressive disorder aged 28.6-50.9 years with or without COPD reported that the adjusted relative risk of vertebral osteoporotic fractures in individuals with COPD was 1.24 (95\% CI, 1.02-1.51) compared with that in the non-COPD patients [6].

Previous studies have also demonstrated a positive relationship between COPD and a higher risk of osteoporotic fractures in the hip $[7,8]$. Reyes et al. reported that an independent association exists between COPD and increased risk of hip OF in Catalonians [8]. However, none of the abovementioned studies had used a prospective design method. A prospective study conducted by Dam et al. to examine the association between COPD or asthma and NOF revealed that male patients with COPD or asthma had $42 \%$ and $164 \%$ greater risk of sustaining non vertebral and vertebral NOF, respectively [9].The association between COPD and NOF in older patients observed in these studies may be attributable to the shared risks between both conditions, such as advanced age, smoking, physical inactivity, osteoporosis, drugs, dementia, alcohol consumption, estrogen deficiency, impaired eyesight, low body weight, dietary calcium deficiency, susceptibility to falling, and uncontrolled inflammation $[6,10]$.

Statin use has been shown to be independently associated with a decreased risk of osteoporotic fractures [11,12]. Statins are effective agents in controlling dyslipidemia and are widely used in the prevention of cardiovascular diseases [13]. In particular, statins may influence bone metabolism by increasing bone formation [14]. It should be noted that osteoporotic fractures in older patients may result from accidents and events such as falls. Patients with COPD have muscle weakness, mobility impairment, and exercise 
intolerance that make them susceptible to falls. An observational cohort study reported a significant association between COPD and increased risk (adjusted OR, 1.12; 95\% CI, 1.01-1.24) of falls compared to that in non-COPD patients [15]There are no guidelines available on the prevention of osteoporotic fractures in older COPD patients.

Only one previous report has developed a five-step clinical approach for preventing fractures in patients with COPD. This five-step clinical approach consists of clinical case finding, risk evaluation, differential diagnosis, treatment, and follow-up. This clinical approach was developed considering the risk factors for fractures, including classical risk factors (older age, low body mass index, personal and family history of fractures, immobility, smoking, alcohol intake, use of glucocorticoids, and increased fall risk) and COPD-specific risk factors (severe airflow obstruction, pulmonary exacerbations, and oxygen therapy). However, this fivestep clinical approach for preventing fractures has not yet been validated. Further research is needed on algorithms to identify patients with COPD at a high risk of osteoporotic fractures and the beneficial effects of preventing osteoporotic fractures in older patients with COPD.

\section{References}

1. Blume SW, Curtis JR (2011) Medical costs of osteoporosis in the elderly Medicare population. Osteoporos Int 22(6): 1835-1844.

2. Johnell 0, Kanis JA (2006) An estimate of the worldwide prevalence and disability associated with osteoporotic fractures. Osteoporosis Int 17(12): 1726-1733.

3. Halbert RJ, Natoli JL, Gano A, Badamgarav E, Buist AS, et al. (2006) Global burden of COPD: systematic review and meta-analysis. Eur Respir J 28(3): 523-532.

4. Romme EA, Geusens P, Lems WF, Rutten EP, Smeenk FW, et al. (2015) Fracture prevention in COPD patients; a clinical 5-step approach. Respir Res 16: 32 .

5. Watanabe R, Tanaka T, Aita K, Hagiya M, Homma T, et al. (2015) Osteoporosis is highly prevalent in Japanese males with chronic obstructive pulmonary disease and is associated with deteriorated pulmonary function. J Bone Miner Metab 33(4): 392-400.

6. Lee SC, Hu LY, Huang MW, Shen CC, Huang WL, et al. (2017) Risk of vertebral fracture in patients diagnosed with a depressive disorder: a nationwide population-based cohort study. Clinics 72(1): 44-50.

7. Kulak CA, Borba VC, Jorgetti V, Dos Reis LM, Liu XS, et al. (2010) Skeletal microstructural abnormalities in postmenopausal women with chronic obstructive pulmonary disease. J Bone Miner Metab Res 25(9): 19311940.

8. Reyes C, Estrada P, Nogues X, Orozco P, Cooper C, et al. (2014) The impact of common co-morbidities (as measured using the Charlson index) on hip fracture risk in elderly men: a population-based cohort study. Osteoporos Int 25(6): 1751-1758.

9. Dam TT, Harrison S, Fink HA, Ramsdell J, Barrett-Connor E (2010) Osteoporotic Fractures in Men (MrOS) Research Group. Bonemineral density and fractures in oldermen with chronic obstructive pulmonary disease or asthma. Osteoporos Int 21(8): 1341-1349.

10. Hsiao PC, Chen TJ, Li CY, Chu CM, Su TP, et al. (2015) Risk factors and incidence of repeat osteoporotic fractures among the elderly in Taiwan: A population-based cohort study. Medicine 94(7): e532.

11.Wiens M, Etminan M, Gill SS, Takkouche B (2006) Effects of antihypertensive drug treatments on fracture outcomes: a meta-analysis of observational studies. J Intern Med 260(4): 350-362.

12. Kwan E, Straus SE (2014) Assessment and management of falls in older people. CMAJ 186(16): E610-E621.

13. Taylor F, Huffman MD, Macedo AF, Moore TH, Burke M, et al. (2013) Statins for the primary prevention of cardiovascular disease. Cochrane Database Syst Rev 1: CD004816.

14. Mundy G, Garrett R, Harris S, Chan J, Chen D, et al. (1999) Stimulation of bone formation in vitro and in rodents by statins. Science 286(5446): 1946-1949.

15. Sibley KM, Voth J, Munce SE, Straus SE, Jaglal SB (2014) chronic disease and falls in community-dwelling Canadians over 65 years old: a population-based study exploring associations with number and pattern of chronic conditions. BMC Geriatrics 14: 22.

16. Felson DT, Sloutskis D, Anderson JJ, Anthony JM, Kiel DP (1991) Thiazide diuretics and the risk of hip fracture. Results from the Framingham study. JAMA 265(3): 370-373.

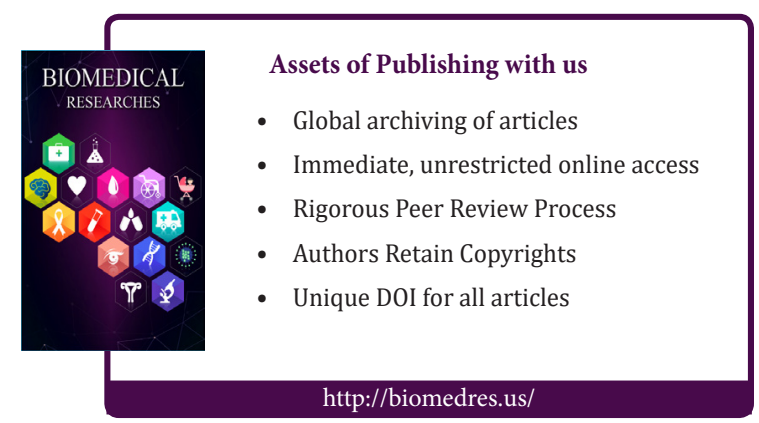

